\title{
Evaluation of the efficacy of EGF and hyaluronic acid formulation on periodontal defects named also black holes
} \author{
Fernández ${ }^{2}$ and Adolfina Ruiz-Martínez ${ }^{1 *}$ \\ ${ }^{1}$ Department of Pharmacy and Pharmaceutical Technology, School of Pharmacy, University of Granada, Granada, Spain \\ ${ }^{2}$ Department of Oral Surgery, School of Dentistry, University of Granada, Granada, Spain \\ ${ }^{3}$ Department of Statistics, University of Granada, Granada, Spain \\ ${ }^{4}$ Department R\&D of Inves Biofarm, Granada Spain
}

Gabriella Russo $^{1}$, Javier Sánchez-Sánchez², Gloria González-Pérez ${ }^{2}$, Ramón Gutiérrez-Sánchez ${ }^{3}$, Rocío Amate-Marchal ${ }^{4}$, Elena Sánchez-

\begin{abstract}
Background: The aim of this study was to determine the efficacy of the application of a formula composed by EGF and Hyaluronic acid 1\% emulsion on periodontal defects or black holes. This clinical trial was approved by the ethical committees in research of University of Granada and carried out according to the Declaration of Helsinki. The study was clinically registered with number 6/CEIH/2015.

Materials and Methods: In this randomized controlled clinical study, data from 23 individuals with chronic periodontitis were evaluated after full-mouth SRP. In the test group $(0)(\mathrm{n}=11)$, EGF-hyaluronic $1 \%$ emulsion was additionally applied during 10 session. The control group $(1)(\mathrm{n}=12)$ was treated with SRP only and hyaluronic acid $1 \%$ gel. The results were statistically examined.

Results: Descriptive variables frequencies were calculated with patient's information. Group 0 sample size was 41 black holes analysed. Difference mean of probing depth was higher at 6 months. Group 1 sample size was 50 black holes. PD $(\mathrm{mm}) \mathrm{I}-3 \mathrm{P}$ is positioned lower than PD I-3P. After 6 months decreasing was higher in both groups than after 3 months. CAL I-6P had a displacement to the bottom respect to I-3P but population median is the same. CAL I-6P of group 1 is more disperse than I-3P. Wilcoxon test was significative when $(\mathrm{P} \leq 0.05)$. Mann-Whitney $\mathrm{U}$-test indicated significance between groups.
\end{abstract}

Conclusion: Both treatments 1\%HA-EGF emulsion and 1\%HA gel increase gingival and bone tissue.

\section{Introduction}

Periodontitis is a chronic periodontal disease associated with plaque biofilms and characterized by periodontium inflammation, gingival bleeding, and presence of periodontal defects or black holes. Inflammatory response affects gingival epithelium, gingival connective tissue, periodontal ligament, alveolar bone and cementum root surface [1]. Diagnosis include loss of periodontal tissue support, clinical attachment loss (CAL), bleeding of probing (BOP), and radiographic bone loss [2]. Systemic diseases and different conditions such as obesity, rheumatoid arthritis, diabetes, smoking or oral hygiene aggravate periodontitis effects [3]. Healthy periodontal tissue has $2 \mathrm{~mm}$ distance from alveolar bone crest (AC) to the cementoenamel junction (CEJ) [4].

Periodontal therapy combined with risk factor control reduce periodontal disease progression. The healing of gingival connective tissue results in a significant reduction of gingival recession and a reduction of the periodontal defects due to generation of new connective tissue attachment 1 . Periodontitis treatment comprise scaling and root planning (SRP) [5], systemic antibiotics [6], and regenerative procedures.

The regenerative procedures aimed to repair the loss of periodontal tissues, including alveolar bone, periodontal ligament and root cementum, are as follows: gum grafts, bone replacement grafts (BRG), root surface biomodification and guided tissue regeneration (GTR). The combination of the use of both methods increase periodontal tissue regeneration $[7,8]$. Novel regenerative procedures include stem cell associated with membranes $[9,10]$.

Hyaluronic acid (HA) is a high-molecular-mass heteropolysaccharide (glycosaminoglycans) [11] found in the extracellular matrix, especially of soft connective tissues. It has structural and physiological function in soft periodontal tissues, gingiva and periodontal ligament, and in the hard tissue, such as alveolar bone and cementum [12]. HA has shown anti-inflammatory and anti-bacterial effects for the treatment of periodontal disease [13,14]. Subgingival $0.8 \% \mathrm{HA}$ gel reduce bleeding after 12 weeks $[15,16]$. Uses of HA gel after scaling and root planning (SRP) treatment improve periodontitis parameters such as bleeding on probing (BOP), plaque index, probing pocket depth (PPD), clinical attachment loss (CAL), and colony-forming units (CFUs) compared with the control site treated with SRP only [17-19].

Recombinant growth factors are known to promote skin and bone wound healing. Pre-clinical and clinical trials use it for the treatment of large periodontal or infra-bony defects [20,21]. Spongy matrix of

*Correspondence to: Adolfina Ruiz Martínez, Department of Pharmacy and Pharmaceutical Technology School of Pharmacy, University of Granada, Granada, Spain, E-mail: adolfina@ugr.es

Key words: chronic periodontitis, periodontal defects, hyaluronic acid, EGFA

Received: February 18, 2020; Accepted: February 24, 2020; Published: February 27,2020 
HA, collagen and EGF is beneficial in treatment of skin defects. EGF is more effective in wound healing than VEGF and HGF [22]. Epidermal growth factor (EGF) plays an influential role in epithelial cells signalling, important for maintaining the homeostatic balance between epithelial cell proliferation and differentiation [23]. It is an osteogenic differentiation enhancer because it is capable of increasing extracellular matrix mineralization [24].

Recent research connects EGF with Porphyromonas gingivalis. P. gingivalis is a pathogen implicated in chronic periodontitis disease [25]. The stimulation of chemokine CXCL14 expression by $P$. gingivalis is reduced by EGF via MEK-ERK1/2 pathway [26]. CXCL14 is involved in inflammatory response and autoimmunity in periodontitis patients. Thus, EGF may play a role in inflammatory response reduction associated with periodontitis.

The objective of present clinical trial is to determinate the healing periodontium effects of EGF combined with HA gel (1\% Hyaluronic acid-EGF emulsion) compared with HA gel without EGF. The main characteristic of the product is its thickness and adhesiveness to improve its time of releasing on gingival tissue. 1\% HA-EGF and $1 \%$ HA gel were applied with electroporation technique. Electroporation improve macromolecules moving to cytoplasm compartment due to cell membrane acts as dielectric capacitor. Potential and dipolar electric field cell membrane is given by phospholipid bilayer, a Maxwell stress leading to pore formation.

\section{Materials and methods}

\section{Study design}

The present randomized pilot clinical was conducted by the University of Granada in compliance with Dental Clinic and Inves Biofarm. It was a double-blind controlled study that enrolled 23 participants. During the evaluation of patients information regarding sex of the subject, alcohol, tobacco, and oral hygiene habits, brushing frequency, mouthwash use, dental floss use, bruxism, diabetes, hypertension, osteoporosis, other systemic diseases, and number of black holes Patient recruitment was carried out during the first six months of the study. Treatment was applied during following five months. The patients attended the Dental Clinic 1 month after last treatment session to evaluate the effects of treatment.

The main outcome of the study was to evaluate the Periodontitis Parameters Assessment.

Three clinical variables of periodontitis were measured: probing depth (PD), bone loss and clinical attachment loss (CAL). Probing depth was determined by PCP-UN-C15 sounding line. CAL was measure with patient's periodontium pictures analysed by Image Manipulation Program GIMP. GIMP calculated black pixel (correspond to periodontal defects or black holes) and convert them to physical measurement. Bone loss was determinate with alveolar crest longitude (ACL) measured with radiographs of patient's periodontium and calculating by Dent-A-View (c) software. PD and CAL data were collected at the beginning, at three months and the end. Bone loss data was only collected at the beginning and at the end.

\section{Ethics}

This clinical trial was approved by the ethical committees in research of University of Granada and carried out according to the Declaration of Helsinki. The study was clinically registered with number 6/CEIH/2015

All volunteers were recruited at Doctor (E.S.F.) Dental Clinic and agreed to participate in the study. Firstly, each participant was informed with an information sheet about the study and signed an informed consent. Patients information and study result were kept in an anonymous computer database. The study included 23 adult patients with periodontal defects: 19 women and 4 men aged 18 to 60 . 11 patients were randomly assigned to group 1 and remaining patients (12) were randomly assigned to group 0.

\section{Inclusion criteria}

- Healthy periodontal patient without biofilm plaque.

- Periodontal defects called also black holes (1 to 8 ) at anterior sector, between canines of both dental arches. It is measured from the point of interdental contact to the gingival margin

- Patients without antibiotic treatment for the past 6 months.

- Controlled periodontitis disease.

\section{Exclusion criteria}

- Treatment compounds allergic patients.

- Drug-therapy patients causes gingival hyperplasia.

- Pregnancy or lactation patients.

- Infectious disease patient such as HIV or Hepatitis C.

\section{Treatment Procedure}

Therapy was implemented by periodontist. Group 1 was treated with $1 \%$ HA gel. Group 0 was treated with $1 \%$ HA-EGF emulsion. After professional tooth cleaning (scaling and root planning (SRP)), 1\% HA gel and 1\% HA-EGF emulsion were introduced into all periodontal black holes using specifically designed electroporation terminal. Electroporation system used was ITD Setem ${ }^{\circledR}$ (TDES). Electroporation program consisting of two phases. The first one, electroporation for 7 minutes. The second one, electroporation for 3 minutes. Treatment consist of 10 session: 1 session each week during 4 weeks, 1 session each two weeks during 2 months and 1 session each month during 2 months.

\section{Statistics}

Descriptive variables analysed were sex, age, use of another product or medicine, tobacco and alcohol consumption, oral hygiene, bruxist, tightener. Outcome variables were PD, ACL and CAL.

Variables were analysed by SPSS v22 software. Statistic descriptive analyses to each experimental variable were done. Mean, standard deviation and median were calculated for all clinical variables. This study was analysed with no parametric test (Wilcoxon test and MannWitney test) due to no normal data and population size. Wilcoxon test was used to compare significance between differences of mean. MannWitney test ( $U$ test) was used to analyse the significance between group 0 and group 1. The null hypothesis of the study is that there no statistically significances in the clinical parameters (CAL, AC longitude and PPD) between the group 1 and group 0 . A level of $\alpha \geq 0.05$ was considered significant.

\section{Results}

Descriptive variables frequencies were calculated with patient's information (Table 1).

Volunteers were positioned in group 0 and group 1 randomly. 8 women and 3 men were included in group 0 and 11 women and 1 man in group 1. Tobacco and alcohol consumption patients were higher in group 0 than in group 1 . Most of patients brushed them teethes three 
Table 1. Descriptive variables frequency

\begin{tabular}{|c|c|c|c|}
\hline \multirow{2}{*}{\multicolumn{2}{|c|}{ Variables }} & \multicolumn{2}{|c|}{ Frequency } \\
\hline & & Group 0 & Group 1 \\
\hline \multicolumn{2}{|c|}{$\mathrm{n}$} & 11 & 12 \\
\hline \multicolumn{2}{|l|}{ Female } & 8 & 11 \\
\hline \multicolumn{2}{|l|}{ Male } & 3 & 1 \\
\hline \multicolumn{2}{|l|}{ Tobacco consumption } & 3 & 1 \\
\hline \multicolumn{2}{|l|}{ Alcohol consumption } & 0 & 1 \\
\hline Oral hygiene & & 2 & 2 \\
\hline \multirow{3}{*}{ Brushed frequency } & Once a day & 0 & 1 \\
\hline & twice a day & 2 & 3 \\
\hline & Three times a day & 9 & 8 \\
\hline \multicolumn{2}{|l|}{ Mouthwash use } & 3 & 1 \\
\hline \multicolumn{2}{|l|}{ Dental floss use } & 10 & 9 \\
\hline \multicolumn{2}{|l|}{ Bruxism } & 3 & 5 \\
\hline \multicolumn{2}{|l|}{ Tightener } & 4 & 6 \\
\hline \multicolumn{2}{|l|}{ Diabetes } & 0 & 0 \\
\hline \multicolumn{2}{|l|}{ Hypertension } & 0 & 0 \\
\hline \multicolumn{2}{|l|}{ Osteoporosis } & 0 & 1 \\
\hline \multicolumn{2}{|l|}{ Other systemic diseases } & 2 & 1 \\
\hline \multirow{8}{*}{ Number of black holes } & 1 Black hole & 1 & 0 \\
\hline & 2 black holes & 1 & 1 \\
\hline & 3 Black holes & 3 & 5 \\
\hline & 4 black holes & 1 & 3 \\
\hline & 5 black holes & 5 & 0 \\
\hline & 6 black holes & 0 & 1 \\
\hline & 7 black holes & 0 & 1 \\
\hline & 8 black holes & 0 & 1 \\
\hline
\end{tabular}

times per day ( 9 patients in group 0 and 8 patients in group 1$)$ and used dental floss (10 patients from group 0 and 9 patients from group 1). Only one patient had osteoporosis involve in periodontitis aggravation in group 1. The other systemic diseases were arthrosis (one patient in group 0), hypothyroidism (one patient in group 0) and multiple sclerosis (one patient in group 1). The majority of patients had between 3 to 5 periodontal defects. In group 0: one patient with one black hole, one patient with 2 black holes and one patient with 4 black holes, three patients with 3 black holes and five patients with 5 black holes. In group 1: one patient with 2 black holes, five patients with 3 black holes, three patients with 4 black holes, one patient with 6 black holes, one patient with 7 black holes and one patient with 9 black holes.

Mean differences of pair-samples for outcome variables (PD, ACL and CAL) were calculated. Pair-samples were considered the differences between initial data and 3 months data (named I-3P), between initial data and 6 months data (named I-6P) and between 3 months data and 6 months data (named I-36P). Probing depth I-3P result for group 0 was $-0.5526 \pm 0.4157 \mathrm{~mm}$. PD I-6P mean was $-0.7949 \pm 0.4690$. PD I-36P mean was $-0.2500 \pm 0.3811$. Group 1 PD I-3P mean was -0.6471 \pm 0.5941 . PD I-6P months was $-0.8824 \pm 0.7719$. PD I-36P mean was $-0.2353 \pm 0.3918$. ACL I-6P mean for group 0 was $0.9962 \pm 2.1551$ and for group 1 was $1.4089 \pm 1.9166$. CAL I-3P for group 0 was -0.2326 \pm 0.3763 and for group 1 was $-0.1159 \pm 0.2569$. CAL I-6P mean was $-0.3171 \pm 0.4686$ and for group 1 was $-0.3466 \pm 0.4373$. And CAL I-36P was $-0.0778 \pm 0.2416(\mathrm{P}=0.062)$ for group 0 and $-0.2306 \pm 0.4304$ for group 1 . Wilcoxon test was significative when $(\mathrm{P} \leq 0.05)$. Mann-Witney test ( $\mathrm{U}$ test) indicated significance between groups. At 6 months group 1 mean was higher than group 0 mean for $\mathrm{PD}(\mathrm{P}=0.539)$, ACL $(\mathrm{P}=0.333)$ and CAL ( $\mathrm{P}=0.880)$ (Table 2).

Group 0 sample size was 41 black holes analysed. For each black hole $\mathrm{PD}, \mathrm{ACL}$ and CAL was measured. Comparing PD ( $\mathrm{mm})$ between initial time and 3 months after treatment and initial time and 6 months after treatment. Difference mean of probing depth was higher at 6 months. I-6P population had a displacement to bottom respect to I-3P. Group 1 sample size was 50 black holes. PD ( $\mathrm{mm})$ I-3P is positioned lower than PD I-3P group 0. I-6P was moving to the bottom comparing with I-3P of group 1. PD decreasing of PD demonstrated with displacement to the bottom of IP-6p. After 6 months decreasing was higher in both groups than after 3 months. In Figure 1, CAL I-6P had a displacement to the bottom respect to I-3P but population median is the same. CAL I-6P of group 1 is more disperse than I-3P (Figure 2).

\section{Discussion}

This study analysed healing periodontitis defects of $1 \%$ HA gel comparing (Group 1) to 1\% HA-EGF emulsion (group 0). The aim is to demonstrate EGF combinate with HA gel increase periodontium healing. HA gel was studied previously as regenerative periodontitis method with different results. Andrea Pilloni, et al. results show there is no statically differences between HA gel after SRP and SRP only periodontitis treatments [18]. Yi Xu, et al. determinate that $0.2 \% \mathrm{HA}$ gel has no improving periodontitis clinical parameters after SRP [16]. Rajan, et al. conclude using of $1 \mathrm{ml}$ of $0.2 \% \mathrm{HA}$ after SRP improve periodontitis defects more than only SRP [17]. Others studios say $0.8 \%$ HA gel improve periodontitis defects after 12 weeks treatment with previous SRP process [27]. One clinical trial using $0.8 \%$ HA gel

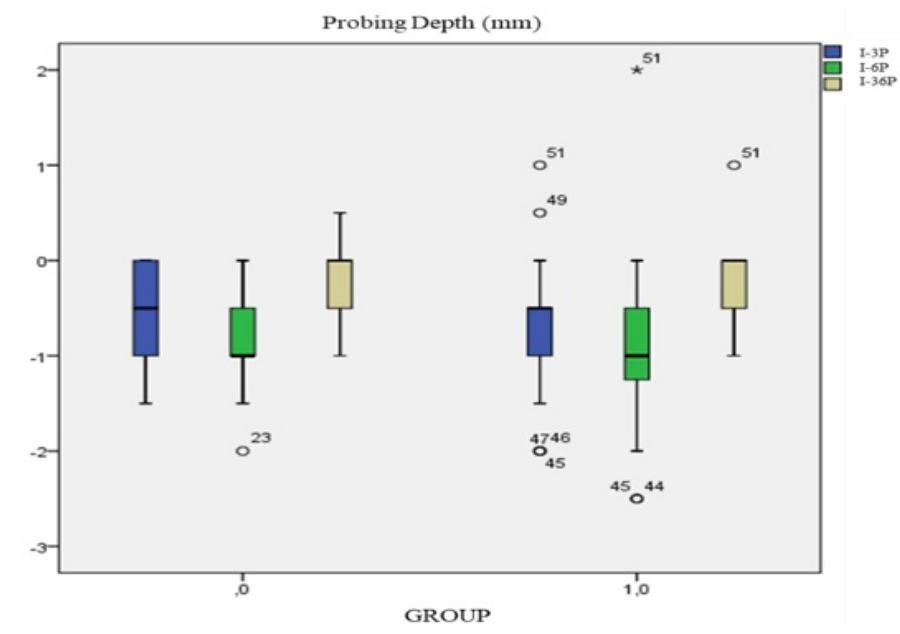

Figure 1. Box plot probing depth

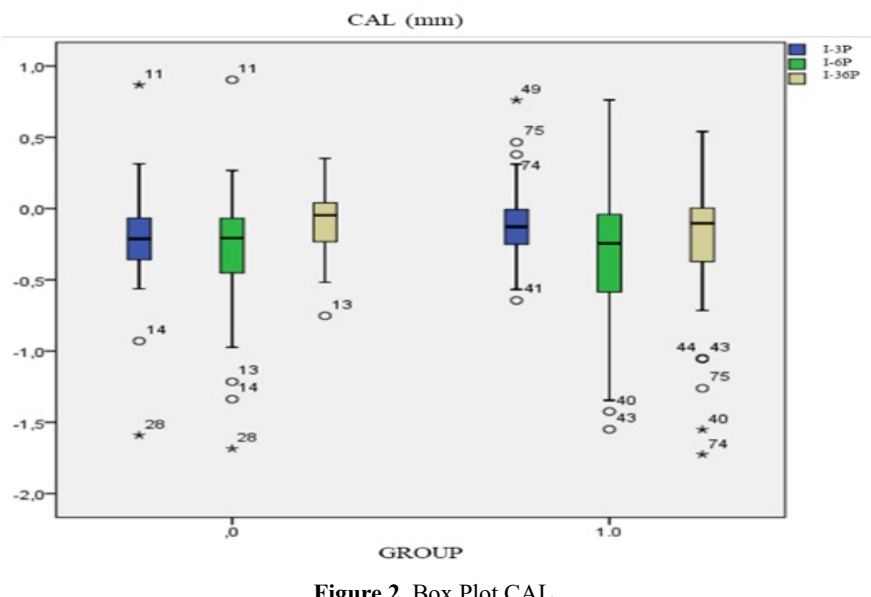

Figure 2. Box Plot CAL 
Table 2. Outcomes variables changes after 3 and 6 months

\begin{tabular}{|c|c|c|c|c|c|}
\hline \multirow{2}{*}{ Outcomes variables } & \multicolumn{2}{|c|}{ Group $0(n=11)$} & \multicolumn{2}{|c|}{ Group $1(n=12)$} & \multirow{2}{*}{$\begin{array}{c}P \\
(\mathrm{U} \text { test })\end{array}$} \\
\hline & Mean \pm SD & $P$ (wilcoxon test) & Mean \pm SD & $P$ (wilcoxon test) & \\
\hline \multicolumn{6}{|c|}{ Probing depth (PD) (mm) } \\
\hline $\mathrm{I}-3 \mathrm{P}$ & $-0.5526 \pm 0.4157$ & 0.000 & $-0.6471 \pm 0.5941$ & 0.000 & 0.451 \\
\hline $\mathrm{I}-6 \mathrm{P}$ & $-0.7949 \pm 0.4690$ & 0.000 & $-0.8824 \pm 0.7719$ & 0.000 & 0.539 \\
\hline $\mathrm{I}-36 \mathrm{P}$ & $-0.2500 \pm 0.3811$ & 0.001 & $-0.2353 \pm 0.3918$ & 0.000 & 0.970 \\
\hline \multicolumn{6}{|c|}{ Alveolar Crest Longitude (ACL) (mm) } \\
\hline $\mathrm{I}-6 \mathrm{P}$ & $0.9962 \pm 2.1551$ & 0.019 & $1.4089 \pm 1.9166$ & 0.000 & 0.333 \\
\hline \multicolumn{6}{|c|}{$\mathrm{CAL}(\mathrm{mm})$} \\
\hline $\mathrm{I}-3 \mathrm{P}$ & $-0.2326 \pm 0.3763$ & 0.000 & $-0.1159 \pm 0.2569$ & 0.000 & 0.880 \\
\hline $\mathrm{I}-6 \mathrm{P}$ & $-0.3171 \pm 0.4686$ & 0.000 & $-0.3466 \pm 0.4373$ & 0.000 & 0.835 \\
\hline $\mathrm{I}-36 \mathrm{P}$ & $-0.0778 \pm 0.2416$ & 0.062 & $-0.2306 \pm 0.4304$ & 0.000 & 0.136 \\
\hline
\end{tabular}

one time and daily $0.2 \%$ HA gel after SRP, after 3 months patients with HA gels improve probing depth parameter scientifically respect control patients [19]. Scaling root planning it is essential process in periodontitis treatment. Present study results demonstrate that higher concentration of hyaluronan (1\% HA gel) during 3 months improve CAL and PD without significally differences between control and test group (Figure 3 and 4 ).

This studio proposes an innovation HA gel contain EGF and ultrasonic process to improve HA penetration. EGF was chosen due to be periodontium proliferation and differentiation enhancer. Recent studio related EGF with periodontitis pathogen P.gingivalis. EGF reduce signally of CXCL14, involve in inflammatory response of $P$. gingivalis in periodontitis disease $[25,26]$.

HA and EGF have been used to treat wound healing. A clinical trial made by $\mathrm{Yu}$, et al. show improvement on skin ulcer and wound healing in epidermis and dermis with sponge sheet of hyaluronan and EGF [28]. Hyemin Kim, et al. formulate a patch type film incorporate HA and EGF facilitate EGF delivery in skin to treat diseases [29]. Study present no statistically difference between HA gel and HA-EGF.

Patients information from both groups (Group 0 and group 1) was collected and analysed. External effects of oral hygiene, healthy life style and systemic diseases in treatments was classified as descriptive variables. Due to population size and distribution (no normal distribution), descriptive variables were no statically analysed. Population was no representative to analysis statistical differences between healthy and unhealthy patients due to only one patient had a systemic disease involve in periodontitis. For each patient number of black holes, probing depth, alveolar crest measure and CAL was measure before treatment. The majority of patients have between 3 and 5 black holes. Initial black holes mean in group 0 was $3,727 \pm 1.4206$ and group 1 mean was 4,167 \pm 1.8504 .

Periodontitis is diagnosticate by clinical parameters such as probing depth (PD), bone loss and clinical attachment loss (CAL). These three parameters were analysed by variation of PD $(\mathrm{mm}), \mathrm{ACL}(\mathrm{mm})$ and CAL $(\mathrm{mm})$ before and after treatments.

PD measure longitude of black hole with a sounding line. PD $(\mathrm{mm})$ mean decreasing indicate the periodontium regeneration. PD decreasing after both treatments. The distance $(\mathrm{mm})$ measured with sounding line from the point of interdental contact to the gingival margin, was tiny after 6 months treatments. Group 1 patients improve $\mathrm{PD}$ with a statistical significance comparing to group 0 at three months and at 6 months $(\mathrm{P}=0.451$ and 0.539$)$.

ACL determinate the longitude of alveolar crest growing. Bone loss is a clinical parameter of periodontitis. In this study, $1 \% \mathrm{HA}$ gel

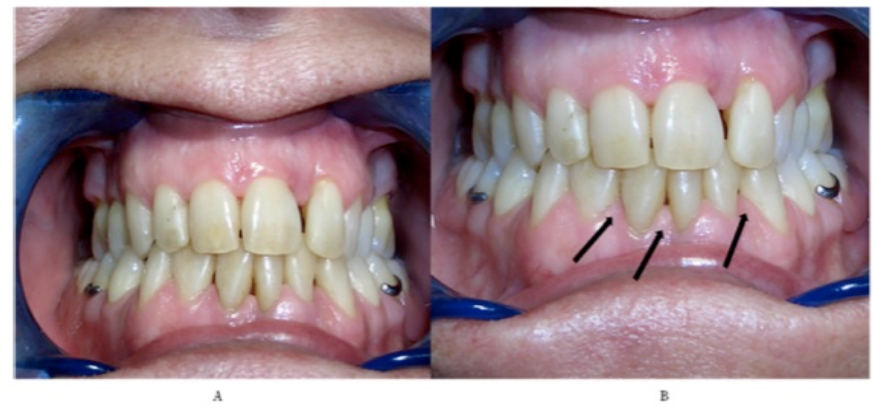

Figure 3. (A) Patient before treatment (B) Patient after 3 months

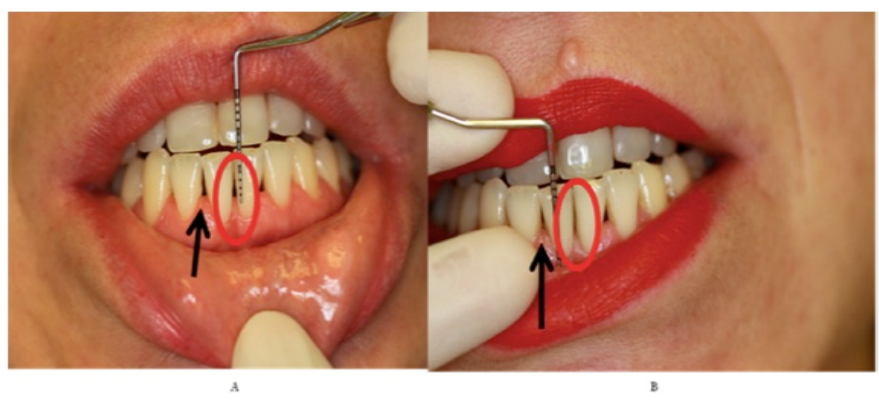

Figure 4. (A) Patient before treatment (B) Patient after 3 months

and 1\%HA-EGF emulsion effects were analysed in bone regeneration. Alveolar crest longitude was measured as indicator of bone growth. After 6 months the measure of ACL was higher than initial data in both treatments. Group 1 patients had higher ACL mean with a significance $(\mathrm{P}=0.33)$ comparing with group 0 .

Clinical attachment loss (CAL) is the area of periodontal black holes. Regeneration of gingival tissue is calculated with decreasing of CAL. Both groups decrease the area of black holes. Group 0 treatment mean is less than group 1 at 6 months with significance. $(\mathrm{P}=0.835)$.

\section{Conclusion}

Both treatments 1\% HA-EGF emulsion and 1\% HA gel increase gingival and bone tissue. EGF have no influence in periodontitis wound healing. HA improve periodontitis defects with $1 \%$ of hyaluronic acid. Further investigation to increase EGF concentration and evaluate effects.

\section{Conflicts of interest}

The authors report no conflicts of interest related to the presented case. The company Inves Biofarm had facilitated the product for this 
study. The study has been carried out in a random and independent way.

\section{References}

1. Ramseier CA, Rasperini G, Batia S, Giannobile WV (2012) Advanced reconstructive technologies for periodontal tissue repair. Periodontol 2000 59: 185-202. [Crossref]

2. Tonetti MS, Greenwell H, Kornman KS (2018) Staging and grading of periodontitis: Framework and proposal of a new classification and case definition. J Clin Periodontol 45 Suppl 20: S149-S161.

3. Albandar JM, Susin C, Hughes FJ (2018) Manifestations of systemic diseases and conditions that affect the periodontal attachment apparatus: Case definitions and diagnostic considerations. J Clin Periodontol 89:183-203.

4. Lang NP, Bartold PM (2018) Periodontal health. J Clin Periodontol 45 Suppl 20: S99S16. [Crossref]

5. Akram Z (2018) How effective is adjunctive antimicrobial photodynamic therapy in treating deep periodontal pockets in periodontal disease? A systematic review. $J$ Investig Clin Dent 9: e12345. [Crossref]

6. Zandbergen D, Slot DE, Cobb CM, Van der Weijden FA (2013) The clinical effect of scaling and root planing and the concomitant administration of systemic amoxicillin and metronidazole: a systematic review. J Periodontol 84: 332-351. [Crossref]

7. Sculean A, Nikolidakis D, Schwarz F (2008) Regeneration of periodontal tissues: combinations of barrier membranes and grafting materials - biological foundation and preclinical evidence: a systematic review. J Clin Periodontol 35: 106-116. [Crossref]

8. Capetillo JF, Coleman BG, Johnson TM (2018) Combination Bone Replacement Graft with Dense Polytetrafluoroethylene Barrier Membrane for Treatment of an Infrabony Periodontal Defect. Clin Adv Periodontics 8: 120-126.

9. Katagiri W, Osugi M, Kawai T, Hibi H (2016) First-in-human study and clinical case reports of the alveolar bone regeneration with the secretome from human mesenchymal stem cells. Head Face Med 12: 5. [Crossref]

10. Chen FM, Gao LN, Tian BM, Zhang XY (2016) Treatment of periodontal intrabony defects using autologous periodontal ligament stem cells: a randomized clinical trial. Stem Cell Res Ther 7: 33. [Crossref]

11. Fallacara A, Baldini E, Manfredini S, Vertuani S. (2018) Hyaluronic acid in the third millennium. Polymers 10: 701.

12. Dahiya P, Kamal R (2013) Hyaluronic Acid: a boon in periodontal therapy. $N$ Am J Med Sci 5: 309-315. [Crossref]

13. Ialenti A, Di Rosa M (1994) Hyaluronic acid modulates acute and chronic inflammation. Agents Actions 43: 44-47. [Crossref]

14. Pirnazar P, Wolinsky L, Nachnani S, Haake S, Pilloni A, et al. (1999) Bacteriostatic effects of hyaluronic acid. J Periodontol 70: 370-374. [Crossref]

15. Al-Shammari NM, Shafshak SM, Ali MS (2018) Effect of $0.8 \%$ Hyaluronic Acid in Conventional Treatment of Moderate to Severe Chronic Periodontitis. J Contemp Dent Pract 19: 527-534. [Crossref]
16. Xu Y, Höfling K, Fimmers R, Frentzen M, Jervøe-Storm PM (2004) Clinical and Microbiological Effects of Topical Subgingival Application of Hyaluronic Acid Gel Adjunctive to Scaling and Root Planing in the Treatment of Chronic Periodontitis. $J$ Periodontol 75: 1114-1118.

17. Rajan P, Baramappa R, Rao NM, Pavaluri AK, P I, et al. (2014) Hyaluronic Acid as an adjunct to scaling and root planing in chronic periodontitis. A randomized clinical trail. J Clin Diagn Res 8: ZC11-14. [Crossref]

18. Pilloni A, Annibali S, Dominici F, Di Paolo C, Papa M, et al. (2011) Evaluation of the efficacy of an hyaluronic acid-based biogel on periodontal clinical parameters. A randomized-controlled clinical pilot study. Ann Stomatol (Roma) 2: 3-9. [Crossref]

19. Eick S, Renatus A, Heinicke M, Pfister W, Stratul SI, et al. (2013) Hyaluronic Acid as an adjunct after scaling and root planing: a prospective randomized clinical trial. $J$ Periodontol 84: 941-949. [Crossref]

20. Fiorellini JP, Howell TH, Cochran D, Malmquist J, Lilly LC, et al. (2005) Randomized study evaluating recombinant human bone morphogenetic protein-2 for extraction socket augmentation. J Periodontol 76: 605-613. [Crossref]

21. Jung RE, Glauser R, Schärer P, Hämmerle CH, Sailer HF, et al. (2003) Effect of rhBMP-2 on guided bone regeneration in humans. Clin Oral Implants Res 14: 556-568. [Crossref]

22. Yu A, Niiyama H, Kondo S, Yamamoto A, Suzuki R, et al. (2013) Wound dressing composed of hyaluronic acid and collagen containing EGF or bFGF: comparative culture study. J Biomater Sci Polym Ed 24: 1015-1026. [Crossref]

23. Parkar MH, Kuru L, Giouzeli M, Olsen I. (2001) Expression of growth-factor receptors in normal and regenerating human periodontal cells. Arch Oral Biol 46: 275-284.

24. Del Angel-Mosqueda C, Gutiérrez-Puente Y (2015) Epidermal growth factor enhances osteogenic differentiation of dental pulp stem cells in vitro. Head Face Med 11: 29. [Crossref]

25. Darveau RP (2010) Periodontitis: a polymicrobial disruption of host homeostasis. Nat Rev Microbiol 8: 481-490. [Crossref]

26. Aw J, Scholz GM, Huq NL, Huynh J, O'Brien-Simpson NM, et al. (2018) Interplay between Porphyromonas gingivalis and EGF signalling in the regulation of CXCL14. Cell Microbiol 20: e12837. [Crossref]

27. Johannsen A, Tellefsen M, Wikesjö U, Johannsen G (2009) Local delivery of hyaluronan as an adjunct to scaling and root planing in the treatment of chronic periodontitis. $J$ Periodontol 80: 1493-1497. [Crossref]

28. Yu A, Takeda A, Kumazawa K, Miyoshi H, Kuroyanagi M, et al (2015) Preliminary Clinical Study Using a Novel Wound Dressing Composed of Hyaluronic Acid and Collagen Containing EGF. Open J Regen Med 4: 6-13.

29. Kim H, Kong WH, Seong KY, Sung DK, Jeong H, et al. (2016) HyaluronateEpidermal Growth Factor Conjugate for Skin Wound Healing and Regeneration. Biomacromolecules 17: 3694-3705. [Crossref]

Copyright: (C2020 Russo G. This is an open-access article distributed under the terms of the Creative Commons Attribution License, which permits unrestricted use, distribution, and reproduction in any medium, provided the original author and source are credited. 